\title{
METÁSTASE CERVICAL DE TUMOR PRIMÁRIO OCULTO: ESTUDO DE 107 CASOS
}

\section{CERVICAL METASTASIS FROM AN UNKNOWN PRIMARY CANCER: STUDY OF 107 CASES}

\author{
Ali Amar ${ }^{1}$ \\ Marcos Ribeiro Magalhães ${ }^{1}$ \\ Abrão Rapoport ${ }^{2}$ TCBC-SP \\ Marcos Brasilino de Carvalho ${ }^{3}$
}

\begin{abstract}
RESUMO: Foram avaliados retrospectivamente 107 pacientes com metástases cervicais de tumor primário oculto, atendidos entre 1977 e 1995 no Hospital Heliópolis. Considerou-se a influência de fatores epidemiológicos e características clínicas na recorrência da doença e sobrevida. O carcinoma epidermóide foi o tipo histológico mais freqüente, acometendo predominantemente as cadeias júgulo-carotídeas alta e média (níveis II e III). Noventa e três por cento dos pacientes apresentavam doença avançada (estadiamento $\mathrm{N}_{2}$ ou $\mathrm{N}_{3}$ ). A sobrevida livre de doença (SLD) aos 5 anos foi de $24 \%$, sendo $40 \%$ para os pacientes com 55 anos ou menos e 8\% para aqueles com mais de 55 anos $(\mathrm{p}=0,01)$. Todos os pacientes com doença na cadeia júgulo-carotídea baixa (nível IV) apresentaram recidiva nos primeiros 12 meses após o tratamento. Os tumores indiferenciados apresentaram recidiva mais precocemente. $\mathrm{Na}$ análise multivariada os fatores idade, tipo de tratamento e estadiamento foram significativos $(\mathrm{p}<0,05)$.
\end{abstract}

Unitermos: Metástases; Tumor primário oculto; Câncer.

\section{INTRODUÇÃO}

A avaliação do significado da linfonodopatia metastática originária de tumor primário oculto persiste como um desafio. Embora muitos tumores sejam diagnosticados no atendimento referenciado, ${ }^{1}$ o sítio primário não é identificado em 2 a $9 \%$ dos pacientes que se apresentam com metástases uni ou bilaterais. ${ }^{2,3}$

Quando diagnosticado nas vias aerodigestivas superiores, geralmente o tumor primário tem origem em locais cujas características anatômicas dificultam a visibilização de lesões iniciais, tais como: hipofaringe, orofaringe (amígdala e base da língua) e rinofaringe.

A metastatização é um indicador de agressividade biológica, mas é a freqüente manifestação de doença regional avançada que sinaliza o mau prognóstico associado a esta condição.

Este trabalho tem por objetivo identificar características epidemiológicas, resultado do tratamento e fatores prognósticos desta condição.

\section{MATERIAL E MÉTODOS}

Foram avaliados os registros de 107 pacientes atendidos no Serviço de Cirurgia de Cabeça e Pescoço do Hospital Heliópolis, no período compreendido entre 1977 e 1995, com queixa de linfonodopatia metastática de tumor primário oculto.

A avaliação diagnóstica inicial incluiu a propedêutica tradicional das vias aerodigestivas superiores, realizada consecutivamente por dois médicos da equipe, com a adição de laringoscopia direta, radiografia de tórax, endoscopia digestiva alta ou esofagograma e tomografia computadorizada.

O tratamento consistiu em cirurgia e radioterapia, isoladamente ou em associação.

Os pacientes foram reestadiados conforme a proposta da UICC-AJC de 1997.

Foram considerados os seguintes fatores: idade, sexo, tabagismo, etilismo, estadiamento TNM, tempo de evolu-

1. Cirurgião do Serviço de Cirurgia de Cabeça e Pescoço do Complexo Hospitalar Heliópolis, São Paulo.

2. Coordenador do Curso de Pós-Graduação em Cirurgia de Cabeça e Pescoço do Complexo Hospitalar Heliópolis, São Paulo.

3. Chefe do Serviço de Cabeça e Pescoço do Complexo Hospitalar Heliópolis, São Paulo.

Recebido em 28/7/99

Aceito para publicação em 10/2/2000

Trabalho realizado no Hospital Heliópolis - São Paulo - SP 
ção da doença, cadeia linfonodal acometida, lateralidade da lesão, histologia e tipo de tratamento.

O diagnóstico histológico empregou cortes corados pela hematoxilina-eosina avaliados à microscopia óptica.

$\mathrm{Na}$ análise de sobrevida foram excluídos 47 pacientes, por não completarem o tratamento ou apresentarem seguimento inferior a 12 meses, sem evidências de recidiva; e também os pacientes com adenocarcinoma ou submetidos à quimioterapia, por não constituírem uma amostra significativa.

A sobrevida livre de doença foi estimada pelo método de Kaplan-Meier ${ }^{4}$. As diferenças observadas entre os subgrupos de cada variável foram avaliados pelo teste de Gehan-Wilcoxon. ${ }^{5}$

A análise multivariada empregou o método de Cox. ${ }^{6}$

A significância estatística foi aceita com erro a inferior a $5 \%$.

\section{RESULTADOS}

Os 107 pacientes com tumor primário oculto correspondem a $5 \%$ dos pacientes com linfonodopatia cervical metastática atendidos no Hospital Heliópolis - São Paulo entre 1977 e 1995.

Noventa pacientes pertenciam ao sexo masculino e 17 ao feminino, constituindo uma relação de aproximadamente 5:1, com idade média de 59,4 \pm 12 anos, sendo $82 \%$ tabagistas e $70 \%$ etilistas.

Não foi observado predomínio de lateralidade da lesão. Em $46 \%$ dos casos acometeu o lado direito, em $43 \%$ o lado esquerdo e em $10 \%$ foi bilateral.

$\mathrm{O}$ intervalo de tempo entre o início dos sintomas e a primeira consulta apresentou média de 5 meses, sem influência na sobrevida livre de doença.

As cadeias júgulo-carotídeas alta e média (níveis II e III) foram as mais atingidas (Figura 1).

O carcinoma epidermóide foi o tipo histológico predominante, especialmente nas formas menos diferencia- das (Figura 2), sendo que $93 \%$ dos pacientes apresentavam doença cervical avançada, com estadiamento $\mathrm{N}_{2}$ ou $\mathrm{N}_{3}$ (Figura 3).

A média etária no grupo que desenvolveu recidiva da doença $(\mathrm{n}=44)$ foi de 59 anos $\left(\mathrm{IC}_{95 \%}=56\right.$ a 61,9), e no grupo livre de doença à ultima consulta $(\mathrm{n}=16)$ foi de 49 $\operatorname{anos}\left(\mathrm{IC}_{95 \%}=42,9\right.$ a 56).

A sobrevida livre de doença (SLD) foi de $24 \%$. Pacientes com idade igual ou inferior a 55 anos apresentaram SLD a cinco anos de $40 \%$, e apenas $8 \%$ dos pacientes com 56 anos ou mais encontravam-se assintomáticos ao término deste período (Gráfico 1). Estes dois grupos foram semelhantes quanto à distribuição dos outros fatores analisados (Tabela 1).

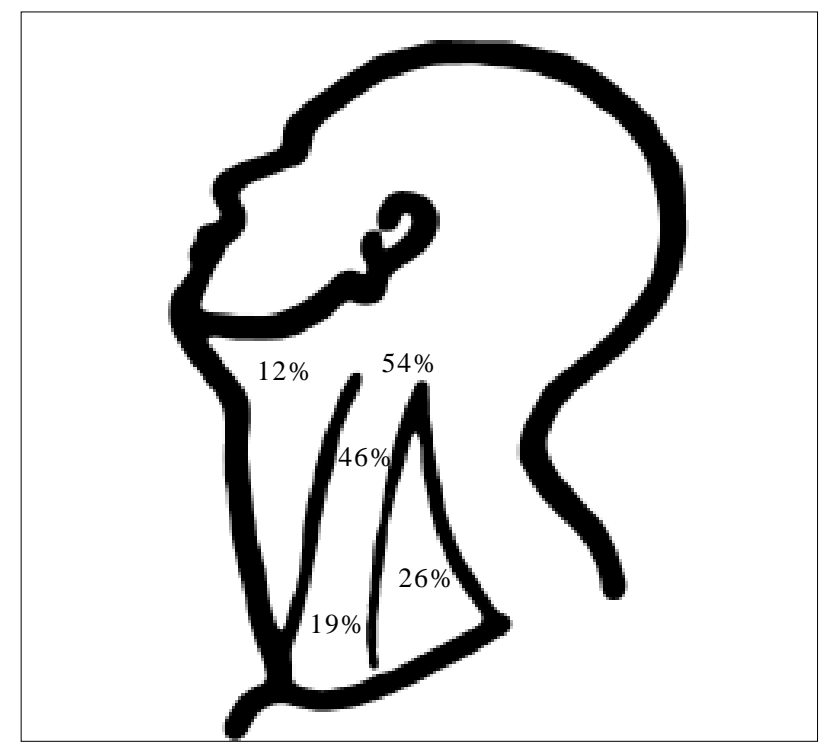

Figura 1 - Distribuição das metástases conforme as cadeias linfonodais (percentual de comprometimento considerando o envolvimento de múltiplas cadeias em um mesmo paciente).

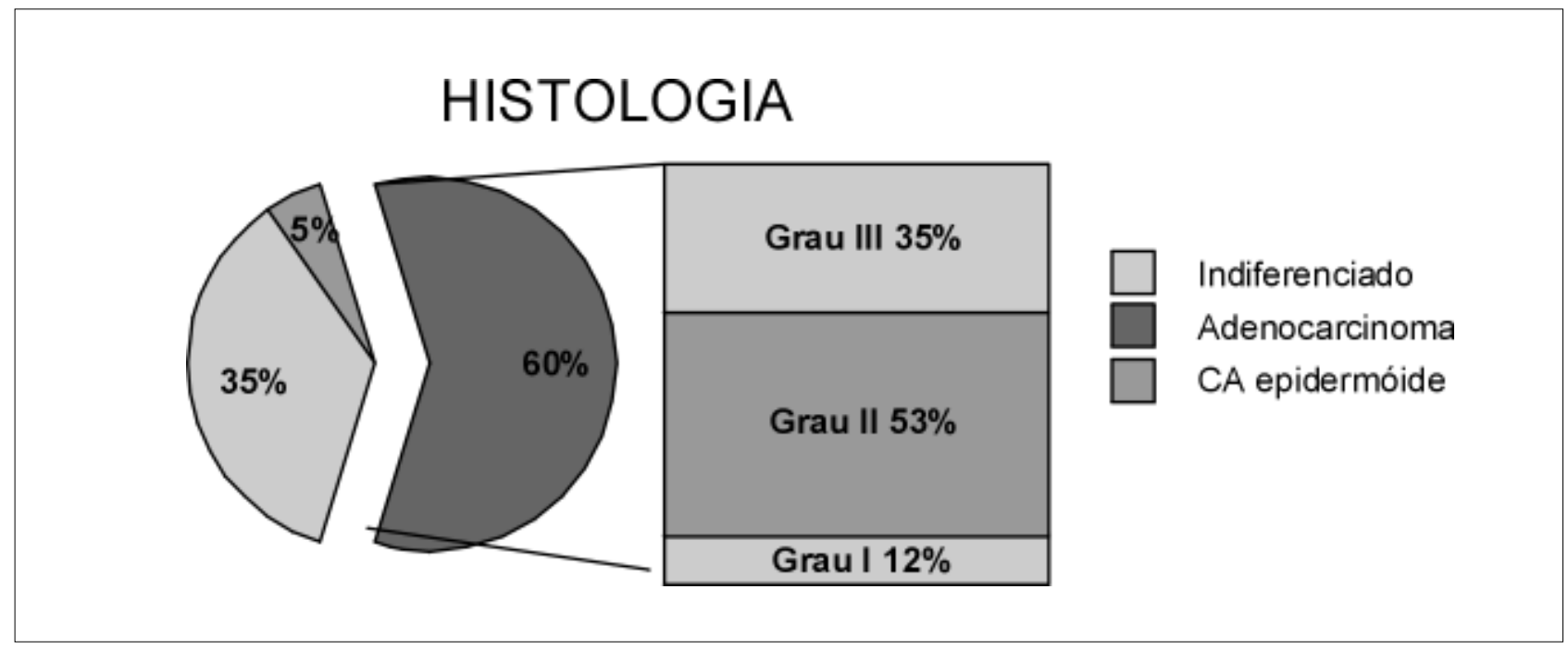

Figura 2 - Distribuição dos pacientes conforme o diagnóstico histológico. 


\section{ESTADIAMENTO}

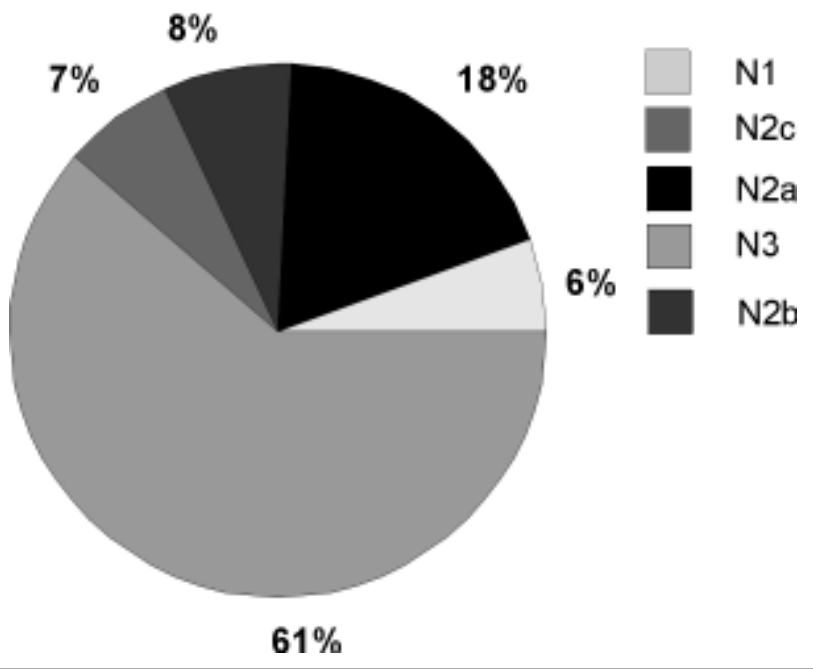

Figura 3 - Distribuição dos pacientes conforme o estadiamento das metástases linfáticas $(N)$.

Tabela 1

Distribuição dos fatores conforme a faixa etária

\begin{tabular}{l|rr}
\hline & \multicolumn{2}{|c}{ Faixa Etária } \\
\cline { 2 - 3 } & $\leq 55$ anos & $>55$ anos \\
\hline Histologia & 3 & 2 \\
I & 10 & 10 \\
II & 8 & 3 \\
III & 6 & 9 \\
Indif. & 3 & 6 \\
NA & & \\
Tratamento & 22 & 20 \\
Cirurgia/Cirurgia + RXT & 8 & 10 \\
Radioterapia & & \\
Estadiamento & 1 & 3 \\
N1 & 5 & 3 \\
N2a & 2 & 1 \\
N2b & 1 & 3 \\
N2c & 18 & 19 \\
N3 & 3 & 1 \\
NA & 30 & 30 \\
\hline Total & & \\
\hline NA & & \\
\hline
\end{tabular}

$N A=$ não avaliado

* Excluídos 47 pacientes não tratados ou com seguimento inferior a 12 meses.

A SLD a cinco anos foi de $28 \%$ para os tumores epidermóides e $18 \%$ para os indiferenciados, sendo que a quase totalidade das recidivas neste último grupo ocorrem nos primeiros seis meses após o tratamento (Gráfico 2).
A SLD foi inversamente proporcional ao estadiamento N (Gráfico 3). Os resultados a cinco anos foram de $75 \%$ para pacientes com estádio $\mathrm{N}_{1}$ e $16 \%$ para $\mathrm{N}_{3}$.

Os pacientes submetidos somente à cirurgia apresentaram resultados semelhantes aos pacientes que receberam radioterapia adjuvante, e ambos mostraram resultados superiores ao tratamento radioterápico exclusivo. Entre os 18 pacientes deste último grupo, 16 evoluíram com persistência da doença ou recidiva precoce (Gráfico 4).

Os nove pacientes com comprometimento de linfonodo júgulo-carotídeo baixo (nível IV) apresentaram recidiva nos primeiros 12 meses após o tratamento.

Seis pacientes $(10 \%)$ desenvolveram doença primária.

$\mathrm{Na}$ análise multivariada, os fatores tratamento, faixa etária e estadiamento $\mathrm{pN}$ foram significativos (Tabela 2).

\section{DISCUSSÃO}

Índices elevados no diagnóstico de tumores primários ocultos podem acusar, mais propriamente, uma propedêutica deficiente. Como estes índices não sofreram variação significativa nas últimas décadas, ${ }^{2,3,7}$ os exames complementares não devem ser superestimados. A tomografia com emissão de pósitrons (PET) pode ser útil na orientação de biópsias, mas apresenta baixa sensibilidade diagnóstica. ${ }^{8}$

A manifestação clínica pode refletir um tumor cujos clones iniciais apresentam elevada capacidade de metastatização. O linfonodo pode constituir um ambiente favorável ao crescimento destes clones, mas provavelmente também desencadeia respostas imunológicas que repercutem no sítio primário. Ronan et al. ${ }^{9}$ 


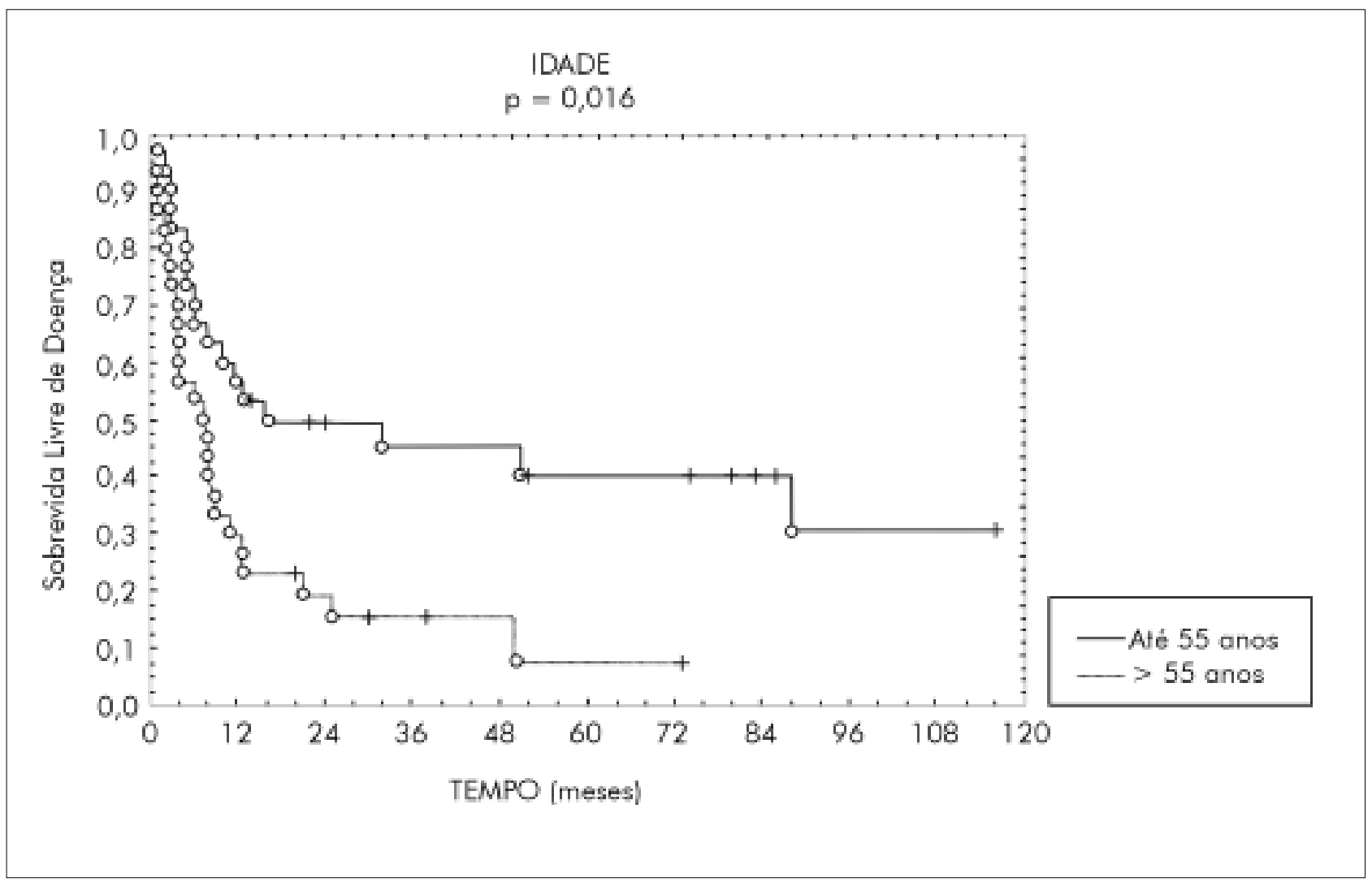

Gráfico 1 - Sobrevida livre de doença conforme a faixa etária.

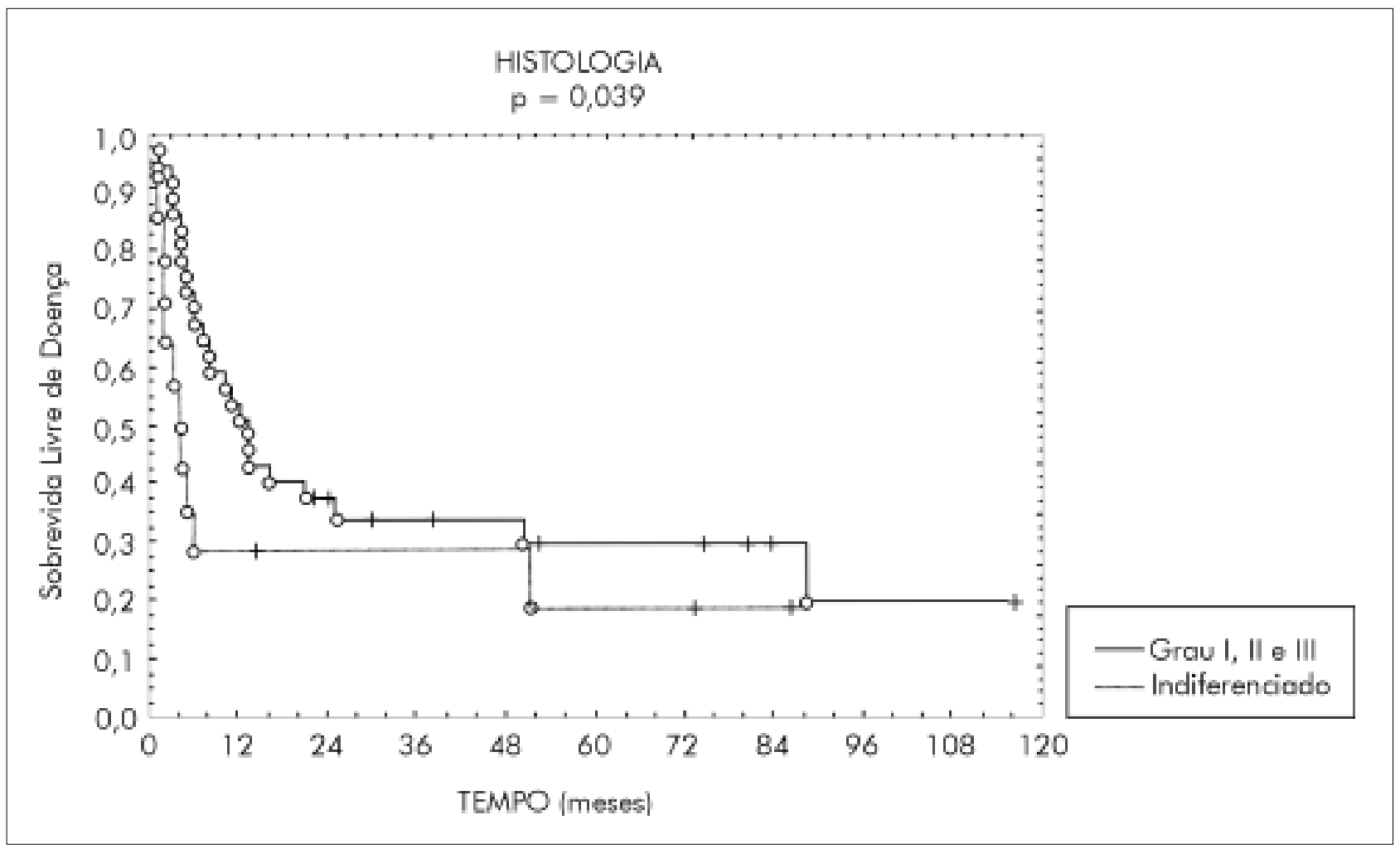

Gráfico 2 - Sobrevida livre de doença conforme o tipo histológico. 


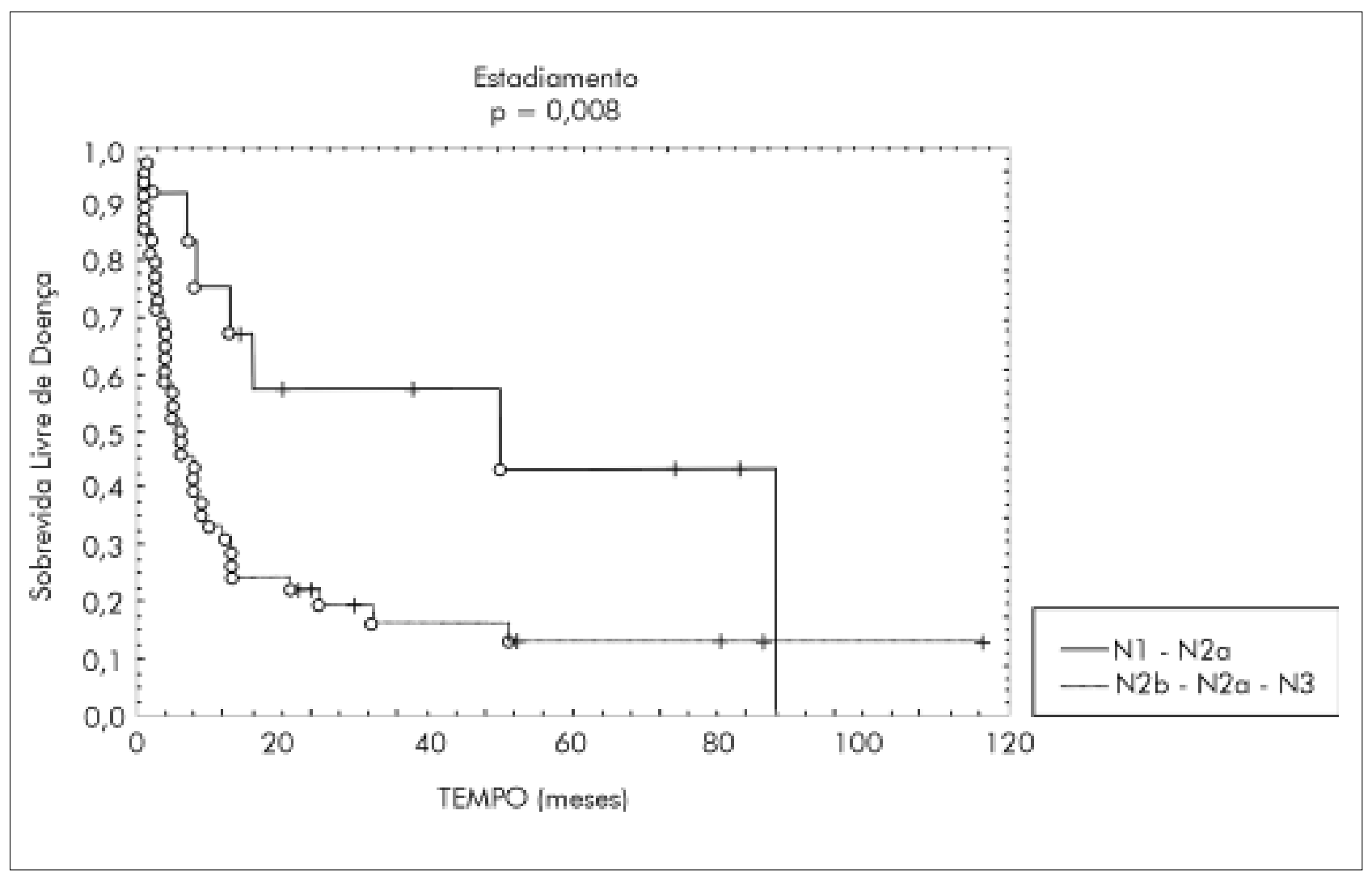

Gráfico 3 - Sobrevida livre de doença conforme o estadiamento $N$.

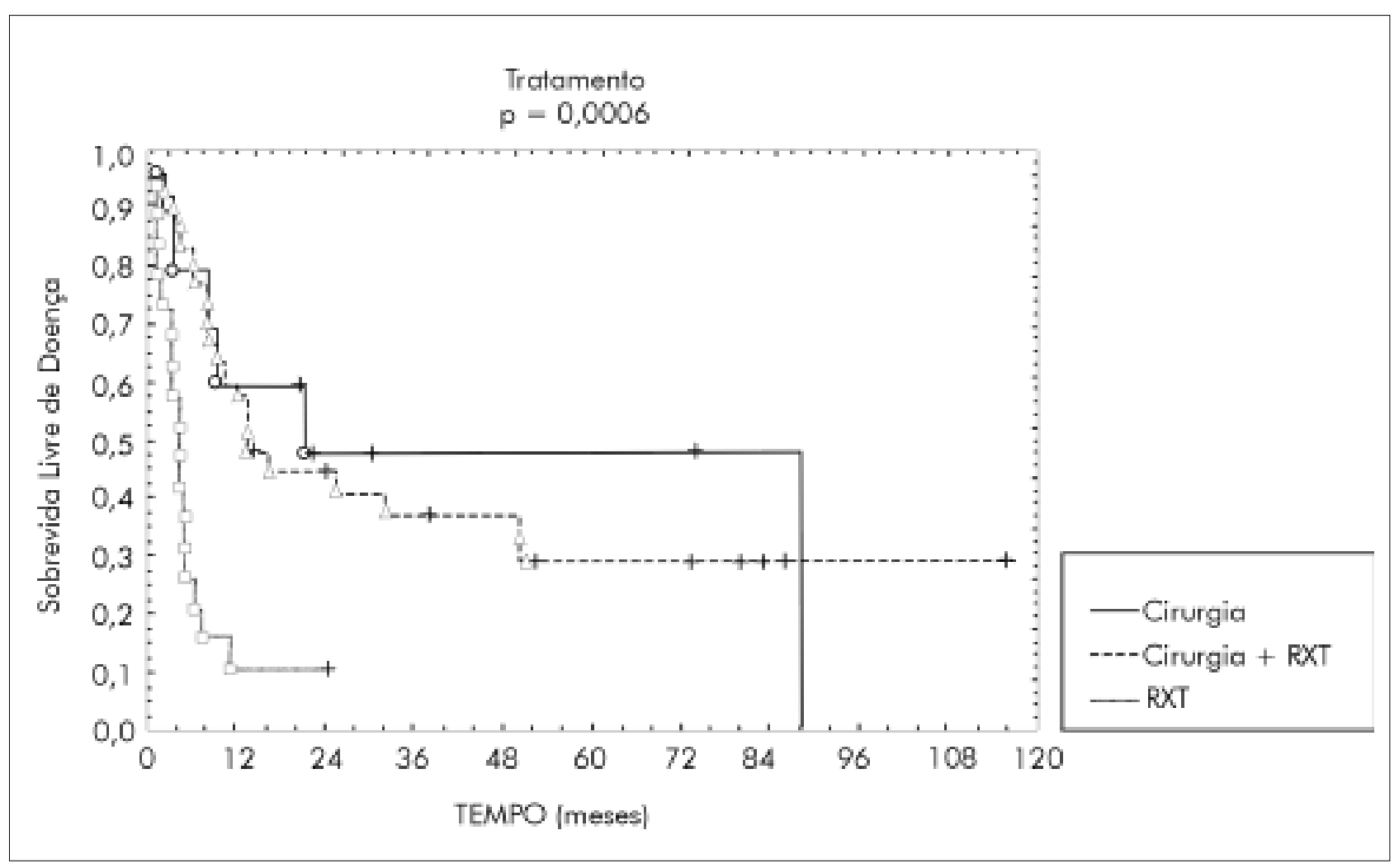

Gráfico 4 - Sobrevida livre de doença conforme o tipo de tratamento. 
Tabela 2

Análise multivariada (Cox)

\begin{tabular}{l|c|c|c}
\hline & $\beta$ & Erro padrão & $p$ \\
\hline Tratamento & 1,086 & 0,319 & 0,0006 \\
Idade & 0,933 & 0,333 & 0,0051 \\
Estadiamento & 0,858 & 0,424 & 0,0428 \\
\hline
\end{tabular}

$\mathrm{Chi}^{2}=25,78$

$p=0,00001$

notaram a regressão local de melanomas na presença de metástases linfonodais. Assim, primários ocultos podem representar tumores que sofreram regressão parcial ou completa em seu sítio de origem. Isto pode explicar o não desenvolvimento de doença primária em alguns pacientes tratados exclusivamente com esvaziamento cervical.

Raramente se desenvolvem carcinomas em cistos branquiais; e apenas poucos casos preenchem os critérios necessários para este diagnóstico. Mais freqüentemente se desenvolvem metástases císticas originárias de tumores da orofaringe. ${ }^{6,10}$

Apenas seis pacientes desenvolveram doença primária, porém não é possível determinar se representam a lesão inicial ou um segundo tumor. Em outras casuísticas foram observados índices entre 7 e $16 \%{ }^{10,11,12}$ de identificação posterior do sítio primário, afetados pela prática de ampliação do campo de irradiação. A incidência de segundo tumor primário em pacientes com carcinoma de cabeça e pescoço é estimada em $4 \%$ ao ano. ${ }^{13}$

Os índices de sobrevida global a cinco anos variam entre 22 e $55 \% .{ }^{14-17}$. No presente estudo a SLD a cinco anos foi de $28 \%$, refletindo tumores com estadiamento avançado na maioria dos pacientes $\left(93 \% \mathrm{~N}_{2}\right.$ e $\left.\mathrm{N}_{3}\right)$, além dos maus resultados obtidos com a radioterapia exclusi- va. Embora este último grupo não seja comparável, a radioterapia, isoladamente, é pouco efetiva no controle da doença cervical avançada. ${ }^{18}$

Medini et al. ${ }^{18}$ apontaram SLD a cinco anos de $14 \%$ para pacientes $\mathrm{N}_{3}$ e $70 \%$ para pacientes $\mathrm{N}_{1-2}$, resultados semelhantes aos encontrados no presente estudo.

A presença de doença extensa, com o envolvimento de múltiplas cadeias, dificulta a avaliação prognóstica do comprometimento linfonodal conforme os critérios anatômicos já estabelecidos. Porém, o envolvimento de cadeias cervicais baixas está fortemente relacionado com a recidiva da doença. ${ }^{19}$ Todos os pacientes com doença nos linfonodos da cadeia IV apresentaram recidiva no primeiro ano pós-tratamento.

O grau de diferenciação dos tumores epidermóides não influiu significativamente nos resultados; a variação observada corresponde provavelmente ao efeito de outros fatores. Os tumores indiferenciados apresentaram recidiva mais precoce, possivelmente relacionada ao menor tempo de replicação.

Os pacientes com idade igual ou inferior a 55 anos apresentaram resultados significativamente melhores, diferentemente do observado em adenocarcinomas ${ }^{20}$ ou carcinomas epidermóides com sítio primário evidente, e confirmado pela análise multivariada. A idade, assim como outros fatores prognósticos, não é um indicador absoluto. A caracterização de uma faixa etária com pior prognóstico sugere a existência de um fenômeno biológico distinto dentro do processo de envelhecimento, talvez relacionado ao sistema imunológico. A eventual não correspondência entre as idades cronológica e biológica não permite a consideração individual dos valores encontrados.

Em conclusão, a idade constitui um fator prognóstico em tumores primários ocultos; o tratamento cirúrgico/ radioterápico é pouco efetivo no controle da doença cervical baixa e os tumores indiferenciados apresentam recidivas precoces.

\begin{abstract}
A retrospective outcome analysis of 107 patients with neck metastasis from an occult primary tumor, was performed at the Head and Neck Service of Heliópolis Hospital, São Paulo, Brazil from 1977 to 1995, being the squamous-cell carcinoma, the more frequent neoplasia. Ninety three percent of patients had advanced disease (stage $\mathrm{N}_{2}$ or $\mathrm{N}_{3}$ ). The 5-year free of disease survival was $24 \%$, forty percent for patients up to 55 years old and $8 \%$ for patients more than 55 years $(p=0,01)$. Patients with undifferentiated tumors and those with disease at level IV had an earlier relapse of disease. The treatment, stage and age were significant in multivariate analysis $(p<0,05)$.
\end{abstract}

Key Words: Cancer; Occult primary; Metastasis.

\section{REFERÊNCIAS}

1. Lee NK, Byers RM, Abbruzzese JL et al - Metastatic adenocarcinoma to the neck from an unknown primary source. Am J Surg 1991, 162:306-309.
2. Comess MS, Beahrs OH, Dockerty MB - Cervical metastases from occult carcinoma. Surg Gynecol Obstet 1957, 104:607-617.

3. Martin H, Morfit HM - Cervical lymph node metastases as the first symptom of cancer. Surg Gynecol Obstet 1944, 78:133-159. 
4. Kaplan EL, Meier P - Nonparametric estimation from incomplete observations. J Am Stat Assoc 1958, 53, 457481.

5. Gehan EA-A generalized Wilcoxon test for comparing arbitrarily singly-censored samples. Biometrika 1965 , 52:650-653.

6. Cox DR - Regression models and life tables. J Royal Stat Soc 1972, 34:187-220.

7. Bouquot JE, Weiland LH, Kurtland LT - Metastases to and from the upper aerodigestive tract in the population of Rochester, Minnesota, 1935-1984. Head Neck 1989, 11:212-218.

8. Kole AC, Nieweg OE, Pruim J et al- Detection of unknown occult primary tumors using positron emission tomografy. Cancer 1998, 82:1160-1166.

9. Ronan SG, Eng AM, Briele HA et al- Thin malignant melanoma with regression and metastases. Arch Dermatol 1987, 123:1326-1330.

10. Maulard C, Housset M, Brunel P et al - Postoperative radiation therapy for cervical lymph node metastases from an occult squamous cell carcinoma. Laryngoscope 1992, 102:884-890.

11. Davidson BJ, Spiro RH, Patel S et al - Cervical metastases of occult origin: the impact of combined modality therapy. Am J Surg 1994, 168:395-399.

12. Glynne-Jones RG, Anand AK, Young TE et al - Metastatic carcinoma in the cervical lymph nodes from an occult primary: a conservative approach to the role of radiotherapy. Int J Radiat Oncol Biol Phys 1990, 18:289294.

13. Hong WK, Lippman SM, Itri LM et al - Prevention of second primary tumors with isotretinoin in squamous-cell carcinoma of the head and neck. N Engl J Med 1990, 323:795-801.
14. Wang RC, Goepfert H, Barber AE et al - Unknown primary squamous cell carcinoma metastatic to the neck. Arch Otolaryngol Head Neck Surg 1990, 116:1388-1393.

15. Fernandez JA, Suarez C, Martinez JA et al - Metastatic squamous cell carcinoma in cervical lymph nodes from an unknown primary tumor: prognostic factors. Clin Otolaryngol 1998, 23:158-163.

16. Reddy SP, Marks JE - Metastatic carcinoma in the cervical lymph nodes from na unknown primary site: results of bilateral neck plus mucosal irradiation vs. Ipsilateral neck irradiation. Int J Radiat Oncol Biol Phys 1997, 37:797-802.

17. Dunst J, Sauer R, Weidenbecher M - Cervical lymph node metastases from an unknown primary tumor. Strahlenther Onkol 1988, 164:129-135.

18. Medini E, Medini AM, Lee CK et al- The management of metastatic squamous cell carcinoma in cervical lymph nodes from an unknown primary. Am J Clin Oncol 1998, $21: 121-125$

19. Jose B, Bosh A, Caldwell WL et al - Metastasis to the neck from an unknown primary tumor. Acta Radiol Oncol Radiat Phys Biol 1979, 18:161-170.

20. Jones AS, Phillips DE, Cook JA et al - Non-squamous malignancy in lymph nodes: the occult primary. Clin Otolaryngol 1993, 18:311-316.

\section{ENDEREÇO PARA CORRESPONDÊNCIA:}

Prof. Abrão Rapoport

Praça Amadeu Amaral, 47/cj. 82

01327-010 - São Paulo-SP 